\title{
A Mechanism for Triple-Ridge Emission Structure of AGN Jets
}

\section{Taiki Ogihara*}

Astronomical Institute, Tohoku University, Sendai, 980-8578, Japan

E-mail: t.ogiharadastr.tohoku.ac.jp

\section{Kazuya Takahashi}

Center for Gravitational Physics, Yukawa Institute for Theoretical Physics, Kyoto University, Kyoto, 606-8502, Japan

\section{Toma Kenji}

Astronomical Institute, Tohoku University, Sendai, 980-8578, Japan

Frontier Research Institute for Interdisciplinary Sciences, Tohoku University, Sendai, 980-8578, Japan

\begin{abstract}
Recent radio very long baseline interferometry (VLBI) observations reveal a triple-ridge emission structure of the relativistic jet in M 87 radio galaxy that consists of the conventional limbbrightened feature and a narrow central ridge. Motivated by these observations, we examine a steady axisymmetric force-free model of a jet driven by the central black hole with its electromagnetic structure being consistent with general relativistic magnetohydrodynamic (GRMHD) simulations, and shown that the model with a velocity field associated with the electromagnetic field and a simple Gaussian distribution of emitting electrons at the jet base can produce the triple-ridge structure. This study will be complementary to theoretical analyses of the future observations of active galactic nucleus (AGN) jets.
\end{abstract}

High Energy Phenomena in Relativistic Outflows VII - HEPRO VII

9-12 July 2019

Facultat de Física, Universitat de Barcelona, Spain

\footnotetext{
* Speaker.
} 


\section{Introduction}

Relativistic jets have been observed in many astrophysical systems such as black hole binaries, gamma-ray bursts, and active galactic nuclei (AGNs). The long-standing problems of AGN jets are its launching mechanism and the injection mechanism.

It has been discussed during many years whether jets are launched by a black hole located

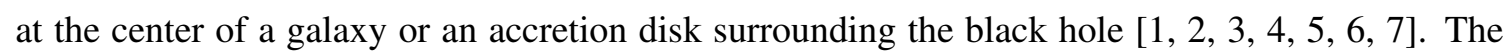
former mechanism is called the Blandford-Znajek (BZ) Process, and the latter one is called the Blandford-Payne (BP) process. Recent sophisticated general relativistic magnetohydrodynamic (GRMHD) simulations show that the globally ordered magnetic field is realized only in the funnel region around the rotation axis. A relativistic jet appears to form via the BZ process, while the disk

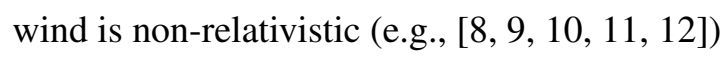

It is not clear how the jetted matter is injected. The globally ordered magnetic field prevents the surrounding thermal plasma particles from diffusing into the jet. In GRMHD simulations, the fluid density in the jet launching region becomes very low and reaches the density floor value, which is set artificially to compute correctly. It is thought that electron-positron pair creation by ambient photons [[13, [4] and by high-energy photons emitted by electrons accelerated in the MHD-violated

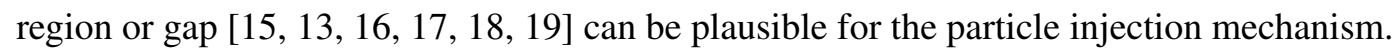

Radio very long baseline interferometry (VLBI) observations can resolve some of the AGN jets. Especially, the jet of M 87 galaxy has been observed to investigate the jet physics due to its proximity. Observations have shown that the M 87 jet has a characteristic emission structure called "the limb-brightened structure," in which the jet edge is brighter than the vicinity of the jet axis [201, 211, 22], 231, 24, 25]]. This structure is considered to be explained by a black hole driven jet model [26]. Slowly spinning black holes and Keplerian disks do not efficiently accelerate the flow. The fluid velocity becomes toroidal dominant, which leads to highly asymmetric images that are not consistent with the observed emission structure.

Recently, high-sensitivity observations with VLBA + phased-VLA [R7] and analysis of VSOP data [28], and stacked VLBA images [29] have revealed the "triple-ridge" emission structure of the M 87 jet that consists of the limb-brightened feature and a narrow central ridge. We focus on this newly discovered emission structure and show that this structure can be explained by the steady axisymmetric force-free jet model, which follows the formulations of [26].

\section{Force-free jet model}

We examined a special relativistic steady axisymmetric force-free jet model in which the electromagnetic field shape is consistent with the results of GRMHD simulations. The poloidal field can be expressed by the magnetic flux function $\Psi$ as

$$
\mathbf{B}_{\mathrm{p}}=\frac{1}{R} \nabla \Psi \times \hat{\phi}
$$

$(r, \theta, \phi)$ is the standard spherical coordinate. $\hat{\phi}$ is the azimuthal unit vector. $R=r \sin \theta$ is the cylindrical radius and $z=r \cos \theta$ is the hight from the equatorial plane. The flux function has been approximated as

$$
\Psi=A r^{v}(1 \mp \cos \theta)
$$


in GRMHD simulations [30, [2], where the plus and minus signs stand for the case of $z<0$ and $z>0$, respectively. $A$ is a normalization factor. $v$ controls the jet shape. When $v=0$, the field lines are the radial shape and when $v=1$, they are parabolic. The toroidal field component is given approximately by [3]

$$
B_{\phi}=\mp \frac{2 \Omega_{\mathrm{F}} \Psi}{R c} .
$$

$c$ is the speed of light and $\Omega_{F}$ corresponds to the angular velocity. The electric field is determined from the ideal MHD condition

$$
\mathbf{E}=-\frac{1}{c} \Omega_{\mathrm{F}} \nabla \Psi=-\frac{R \Omega_{\mathrm{F}}}{c} \hat{\phi} \times \mathbf{B} .
$$

In a force-free model, we cannot determine the velocity field of the fluid. We assume it as the drift velocity which is consistent with the velocity in the Poynting flux dominated cold MHD jet model

$$
\mathbf{v}=c \frac{\mathbf{E} \times \mathbf{B}}{B^{2}}=R \Omega_{\mathrm{F}} \hat{\phi}-R \Omega_{\mathrm{F}} \frac{B_{\phi}}{B^{2}} \mathbf{B} .
$$

The fluid density $n$ follows the continuity equation,

$$
\nabla \cdot(n \mathbf{v})=0
$$

and the density of the emitting electrons is set to a certain fraction of the fluid density. The parameters $R_{p}, z_{1}$ and $\Delta$ determine the density at the jet base.

$$
n\left(R, z_{1}\right)=n_{0} \exp \left(-\frac{\left(R-R_{p}\right)^{2}}{2 \Delta^{2}}\right)
$$

where $n_{0}$ is the normalization factor. The continuity equation and the ideal condition leads to

$$
\frac{n}{B^{2}}=\text { constant along the field lines. }
$$

The energy distribution of the electrons are set to a single power-law spectrum.

$$
f\left(\gamma^{\prime}\right) \propto \begin{cases}\gamma^{\prime-p} & \text { for } \gamma^{\prime}>\gamma_{m}^{\prime} \\ 0 & \text { for } \gamma^{\prime}<\gamma_{m}^{\prime}\end{cases}
$$

where $\gamma^{\prime}$ is the Lorentz factor of electrons measured in the fluid frame, $\gamma_{m}^{\prime}$ is the minimum value of $\gamma^{\prime}$, and $p$ is the power-law index.

We assumed that the radiation is the synchrotron emission from electrons. The emissivity in the fluid frame is

$$
j_{\omega^{\prime}}^{\prime}\left(\hat{\mathbf{n}}^{\prime}\right)=\frac{\sqrt{3}(p-1) e^{3} n^{\prime} B^{\prime} \sin \alpha^{\prime}}{8 \pi^{2} \gamma_{m}^{\prime}{ }^{1-p} m_{e} c^{2}(p+1)}\left(\frac{m_{e} c \omega^{\prime}}{3 e B^{\prime} \sin \alpha^{\prime}}\right)^{-\frac{p-1}{2}} \bar{\Gamma}\left(\frac{p}{4}+\frac{19}{12}\right) \bar{\Gamma}\left(\frac{p}{4}-\frac{1}{12}\right)
$$

from [32], where $e, m_{e}$, and $\bar{\Gamma}(x)$ are the elementary charge, the mass of electron, and the gamma function, respectively. $B^{\prime} \equiv\left|\mathbf{B}^{\prime}\right|$, and $\alpha^{\prime}$ is the pitch angle of the electrons defined by $\cos \alpha^{\prime}=$ $\left(\hat{\mathbf{n}}^{\prime} \cdot \mathbf{B}^{\prime}\right) /\left|\mathbf{B}^{\prime}\right|$, where $\hat{\mathbf{n}}^{\prime}$ is the unit vector directing toward the observer in the fluid frame. After the Lorentz transformation, the emissivity in the observer's frame is

$$
j_{\omega}(\hat{\mathbf{n}})=\frac{1}{\Gamma^{2}(1-\beta \mu)^{3}} j_{\omega^{\prime}}^{\prime}\left(\hat{\mathbf{n}}^{\prime}\right),
$$


The intensity is calculated in the optically thin limit.

$$
I_{\omega}(X, Y)=\int j_{\omega}(\hat{\mathbf{n}}, X, Y, Z) d Z
$$

This model was also used in [26]. They showed that this model could reproduce limbbrightened jet images with the ring-like density distribution at the jet base $\left(R_{\mathrm{p}}>0\right)$. We changed the parameter values and showed that the triple-ridge structure is also obtained with this model.

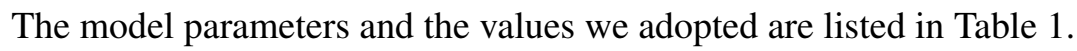

Table 1: Model parameters

\begin{tabular}{llr}
\hline Quality & symbol & value \\
\hline \hline Mass of the black hole & $M_{\mathrm{BH}}$ & $6.2 \times 10^{9} M_{\odot}$ \\
Dimensionless Kerr parameter of the BH & $a$ & 0.998 \\
Rotational frequency of the magnetic field & $\Omega_{\mathrm{F}}$ & $0.5 \Omega_{\mathrm{H}}$ \\
Viewing angle & $\Theta$ & $R_{\mathrm{p}}$ \\
Jet Shape (Eq. [2.2) & $z_{1}$ & $\Delta$ \\
Radius where $n$ peaks at $z= \pm z_{1}$ & $p$ & $16.7 \mathrm{Mpc}$ \\
Height of the plane where $n$ is given & $\gamma_{\mathrm{m}}^{\prime}$ & $16^{\circ}$ \\
Width of $n$ distribution & $D$ & $2 R_{\mathrm{S}}$ \\
Energy spectral index of the non-thermal electrons & & 1.1 \\
Minimal Lorentz factor of the non-thermal electrons & & 1.14 mas $\times 0.55 \mathrm{mas}$ \\
Luminosity distance to the jet & & \\
Inclination between the jet axis and the major axis of the beam kernel \\
Beam size
\end{tabular}

\section{Results}

We produced computed images with the parameters consistent with the observations and simulations for the M 87 jet. Figure $\mathbb{U}$ shows the intensity profiles with different parameter values at 25 mas from the black hole in the projected coordinate. The emission structure is susceptible to the model parameters and the viewing angle $\Theta$. We note that these profiles are normalized by the value at the jet axis $(X=0)$ of each line.

The outer ridges, which are a conventional limb-brightened component, are produced because the magnetic field is stronger, and the density is larger compared to the ones near the jet axis. Despite the weak magnetic field and the low density, the inner ridge is produced due to the relativistic beaming effect. The magnetic field is poloidal dominant near the jet axis and toroidal dominant in the far region. The fluid velocity in our model is perpendicular to the magnetic field. There is a region in which the velocity becomes parallel to the line of sight. This beaming produces the strong emissivity in the observer's frame and the inner ridge component of the jet.

The upper left panel of Figure $\amalg$ shows the intensity profiles in the case of $\Theta=7^{\circ}, 10^{\circ}, 15^{\circ}, 20^{\circ}, 25^{\circ}$. For smaller $\Theta, \mu$ in the accelerated region, which consists of the outer ridges, becomes close to unity, which leads to the less clear inner ridge. The upper right panel of Figure $\square$ shows the intensity profiles in the case of $v=0.7,0.75,0.8 . v=0.75$ is the proposed value by the observations 

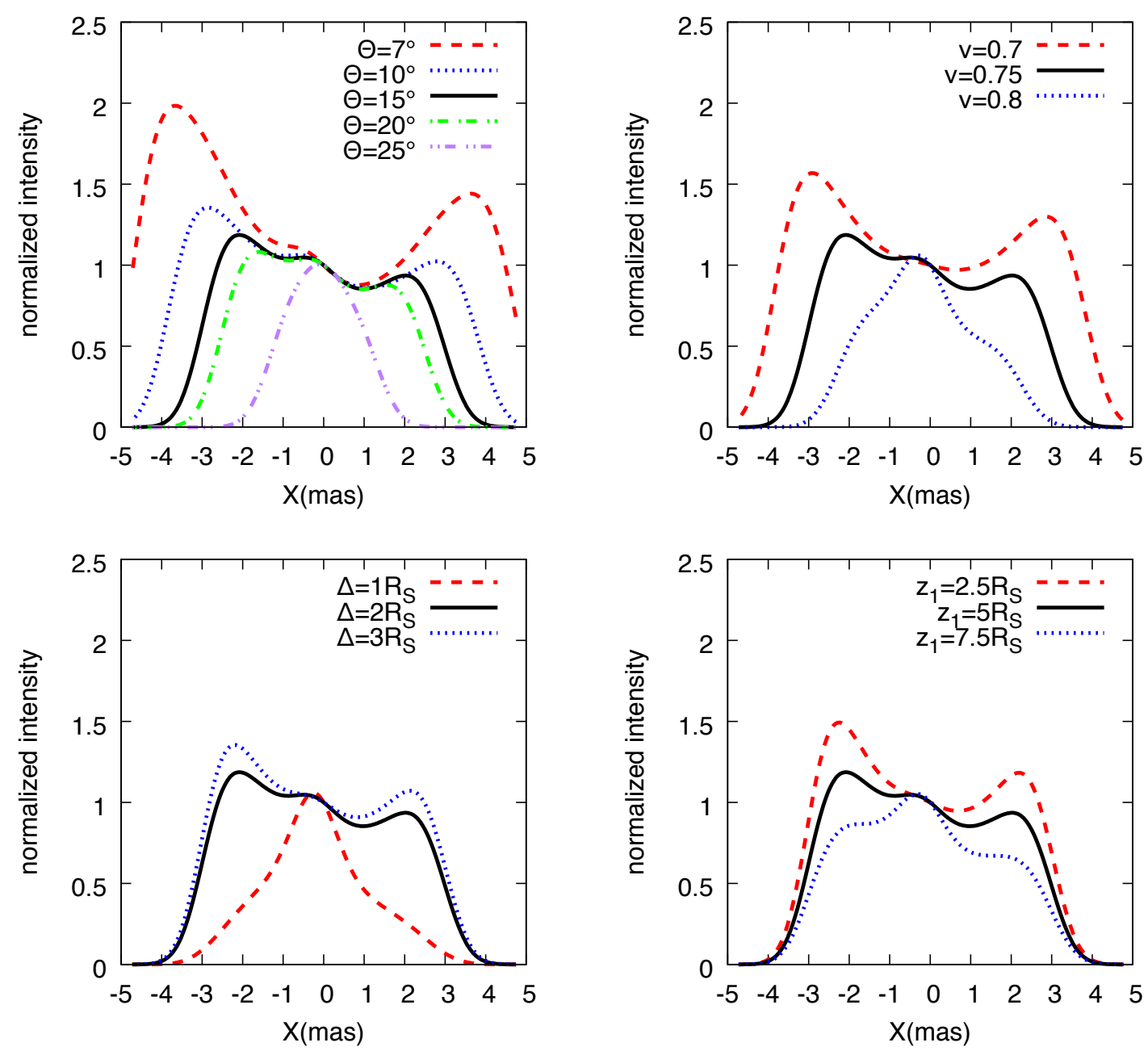

Figure 1: Dependence of the transverse intensity profile at $Y=25$ mas on $\Theta$ (upper left), $v$ (upper right), $\Delta$ (lower left), and $z_{1}$ (lower right) from [B3]. The solid line in each panel shows the result with our fiducial parameters listed in Table $\mathbf{\square}$. The dashed, solid, dotted, dashed-dotted, and dashed double-dotted lines represent the results calculated with changing the parameter values from the fiducial ones. The vertical axis is normalized by the value at $X=0$ of each line.

and numerical calculations [12]. For smaller $v$, the jet becomes wide, and the outer ridges become dominant. The bottom left panel and the bottom right panel of Figure $\square$ show the intensity profiles in the case of $\Delta=1 R_{\mathrm{S}}, 2 R_{\mathrm{S}}, 3 R_{\mathrm{S}}$ and $z_{1}=2.5 R_{\mathrm{S}}, 5 R_{\mathrm{S}}, 7.5 R_{\mathrm{S}}$, respectively. These parameters are related to the density distribution. As $\Delta$ increases or $z_{1}$ decreases, the density in the jet edge become larger and the outer ridges become brighter. Although our setup for the electron density distribution is a toy model, our results indicate that the observed emission structure can strongly constrain the spatial electron density distribution when $\Theta$ and $v$ are estimated by other observational information such as the blob pattern speed, the brightness ratio between the approaching and counter jets, and the width profile of the jet.

Our results and discussion are contained in our more detailed companion paper [B3]]. 
We thank the participants in the Mizusawa Project Meetings in 2016, 2017, and 2018 for fruitful discussions on relativistic jets. Numerical calculations were performed on Draco, a computer cluster of the Frontier Research Institute for Interdisciplinary Sciences in Tohoku University. T.O. acknowledges financial support from Graduate Program on Physics for the Universe (GP-PU), Tohoku University. This work is partly supported by JSPS Grants-in-Aid for Scientific Research 17H06362 (K. Takahashi), 18H01245 (K. Toma), and also by a JST grant Building of Consortia for the Development of Human Resources in Science and Technology (K. Toma).

\section{References}

[1] R. D. Blandford and R. L. Znajek, Electromagnetic extraction of energy from kerr black holes, Monthly Notices of the Royal Astronomical Society 179 (1977) 433.

[2] R. D. Blandford and D. G. Payne, Hydromagnetic flows from accretion discs and the production of radio jets, Monthly Notices of the Royal Astronomical Society 199 (1982) 883.

[3] Y. Uchida and K. Shibata, Magnetodynamical acceleration of co and optical bipolar flows from the region of star formation, Publications of the Astronomical Society of Japan 37 (1985) 515.

[4] R. V. E. Lovelace, J. C. L. Wang and M. E. Sulkanen, Self-collimated electromagnetic jets from magnetized accretion disks, The Astrophysical Journal 315 (1987) 504.

[5] D. L. Meier, Magnetohydrodynamic production of relativistic jets, Science 291 (2001) 84.

[6] S. S. Komissarov, General relativistic magnetohydrodynamic simulations of monopole magnetospheres of black holes, Monthly Notices of the Royal Astronomical Society 350 (2004) 1431.

[7] V. S. Beskin, Magnetohydrodynamic models of astrophysical jets in memory of vitaly lazarevich ginzburg, Physics-Uspekhi 53 (2010) 1197.

[8] J. C. McKinney and C. F. Gammie, A measurement of the electromagnetic luminosity of a kerr black hole, The Astrophysical Journal 611 (2004) 977.

[9] J. C. McKinney and R. D. Blandford, Stability of relativistic jets from rotating, accreting black holes via fully three-dimensional magnetohydrodynamic simulations, Monthly Notices of the Royal Astronomical Society: Letters 394 (2009) L126.

[10] A. Tchekhovskoy, R. Narayan and J. C. McKinney, Efficient generation of jets from magnetically arrested accretion on a rapidly spinning black hole, Monthly Notices of the Royal Astronomical Society: Letters $418(2011)$ L79.

[11] A. Sądowski, R. Narayan, R. Penna and Y. Zhu, Energy, momentum and mass outflows and feedback from thick accretion discs around rotating black holes, Monthly Notices of the Royal Astronomical Society $436(2013) 3856$.

[12] M. Nakamura, K. Asada, K. Hada, H.-Y. Pu, S. Noble, C. Tseng et al., Parabolic jets from the spinning black hole in $\mathrm{m87}$, The Astrophysical Journal $\mathbf{8 6 8}(2018) 146$.

[13] A. Levinson and F. Rieger, Variable tev emission as a manifestation of jet formation in m87?, The Astrophysical Journal 730(2011) 123.

[14] M. Mościbrodzka, C. F. Gammie, J. C. Dolence and H. Shiokawa, Pair production in low-luminosity galactic nuclei, The Astrophysical Journal 735 (2011) 9.

[15] K. Hirotani and I. Okamoto, Pair plasma production in a force-free magnetosphere around a supermassive black hole, The Astrophysical Journal 497 (1998) 563. 
[16] A. E. Broderick and A. Tchekhovskoy, Horizon-scale lepton acceleration in jets: Explaining the compact radio emission in m87, The Astrophysical Journal 809(2015) 97.

[17] K. Hirotani, H.-Y. Pu, L. C.-C. Lin, H.-K. Chang, M. Inoue, A. K. H. Kong et al., Lepton acceleration in the vicinity of the event horizon: High-energy and very-high-energy emissions from rotating black holes with various masses, The Astrophysical Journal 833 (2016) 142.

[18] K. Ptitsyna and A. Neronov, Particle acceleration in the vacuum gaps in black hole magnetospheres, Astronomy \& Astrophysics $\mathbf{5 9 3}(2016)$ A8.

[19] A. Levinson and N. Segev, Existence of steady gap solutions in rotating black hole magnetospheres, Physical Review D96 (2017).

[20] Y. Y. Kovalev, M. L. Lister, D. C. Homan and K. I. Kellermann, The inner jet of the radio galaxy m87, The Astrophysical Journal $668(2007)$ L27.

[21] R. C. Walker, C. Ly, W. Junor and P. J. Hardee, A vlba movie of the jet launch region in m87, Dournal of Physics: Conference Series 131 (2008) 012053.

[22] K. Hada, A. Doi, M. Kino, H. Nagai, Y. Hagiwara and N. Kawaguchi, An origin of the radio jet in $m 87$ at the location of the central black hole, Nature 477 (2011) 185.

[23] K. Hada, M. Kino, A. Doi, H. Nagai, M. Honma, K. Akiyama et al., High-sensitivity 86 ghz (3.5 mm) vlbi observations of m87: Deep imaging of the jet base at a resolution of 10 schwarzschild radii, The Astrophysical Journal 817 (2016) 131.

[24] F. Mertens, A. P. Lobanov, R. C. Walker and P. E. Hardee, Kinematics of the jet in $m 87$ on scales of 100-1000 schwarzschild radii, Astronomy \& Astrophysics 595 (2016) A54.

[25] J.-Y. Kim, T. P. Krichbaum, R.-S. Lu, E. Ros, U. Bach, M. Bremer et al., The limb-brightened jet of $m 87$ down to the 7 schwarzschild radii scale, Astronomy \& Astrophysics 616 (2018) A188.

[26] K. Takahashi, K. Toma, M. Kino, M. Nakamura and K. Hada, Fast-spinning black holes inferred from symmetrically limb-brightened radio jets, The Astrophysical Journal $\mathbf{8 6 8 ( 2 0 1 8 ) 8 2 .}$

[27] K. Hada, The structure and propagation of the misaligned jet m87, Galaxies 5(2017)2.

[28] K. Asada, M. Nakamura and H.-Y. Pu, Indication of the black hole powered jet in m87 by vsop observations, The Astrophysical Journal 833 (2016) 56.

[29] R. C. Walker, P. E. Hardee, F. B. Davies, C. Ly and W. Junor, The structure and dynamics of the subparsec jet in $m 87$ based on 50 vlba observations over 17 years at 43 ghz, The Astrophysical Dournal $855(2018) 128$.

[30] A. Tchekhovskoy, R. Narayan and J. C. McKinney, Black hole spin and the radio loud/quiet dichotomy of active galactic nuclei, The Astrophysical Journal 711 (2010) 50 .

[31] A. Tchekhovskoy, J. C. McKinney and R. Narayan, Simulations of ultrarelativistic magnetodynamic jets from gamma-ray burst engines, Monthly Notices of the Royal Astronomical Society $\mathbf{3 8 8}(2008)$ 55].

[32] G. B. Rybicki and A. P. Lightman, Radiative Processes in Astrophysics. Weinheim: Wiley-VCH, 1986.

[33] T. Ogihara, K. Takahashi and K. Toma, A mechanism for the triple-ridge emission structure of agn jets, The Astrophysical Journal 877 (2019) 19. 